\title{
Adaptive strategy changes as a function of task demands: a study of car drivers
}

\author{
Fonie Cnossent*, Theo Meimmant and Talib Rothengatter $\$$ \\ $\uparrow$ Artificial Intelligence, University of Groningen, The Netherlands \\ tExperimental and Work Psychology, University of Groningen, The Netherlands
}

Keywords: Driving behaviour; Task demands; Mental effort; Strategies; Task priorities.

\begin{abstract}
When drivers perform additional tasks while driving, research shows conflicting results: primary driving performance may deteriorate but adaptive changes such as reducing driving speed have also been noted. We hypothesized that the nature of the secondary task may be important: drivers may give more priority to tasks that serve goals of the driving task itself, for example route finding, than tasks not directly relevant for driving, for example tuning the radio. The main objective of the present driving simulator study was to test this hypothesis. Twenty subjects performed two different subsidiary tasks while driving through two levels of traffic density: a working memory (WM) task and a map reading (MAP) task. It was hypothesized that in high task demand situations, the WM task, irrelevant for the driving task, would be neglected more than the MAP task. The results confirmed the hypothesis: in MAP conditions, the WM task was indeed neglected, but map reading resulted in more swerving, indicating that the subjects looked at the map despite the high task demands. It is concluded that drivers will be highly motivated to get route information, and RG systems should therefore present their information in a readily understandable format.
\end{abstract}

\section{Introduction}

One of the key features of complex dynamic hierarchical tasks is that the people who perform them have to adapt their behaviour to the dynamic environment. In complex dynamic tasks, operators are usually performing several subtasks at the same time, which all serve different task (sub)goals. Operators have to switch between these task (sub)goals, taking the appropriate action at the appropriate time. This allocation of effort between task goals is a key aspect of the effectiveness of task performance in complex dynamic tasks (Bainbridge 1997). The dynamic adaptation serves to keep task behaviour goal-directed and coherent. The relevant task goal has to be preserved and protected against disturbance. Effective task performance involves selecting relevant information while ignoring irrelevant information. Operators only select information relevant for achieving the current task goal (Vicente and Wang 1998).

The same task performance can be achieved by applying different working methods, and each working method may require a different amount of 'mental work'

*Author for correspondence. e-mail: f.cnossen@ppsw.rug.nl 
(Bainbridge 1974, Welford 1978). When operators are confronted with increased task demands, they can either invest more effort, or adjust the performance targets. In the latter case, operators may decrease the desired level of accuracy or speed; use less demanding strategies; or ignore subsidiary activities (Hockey 1997). In general, operators will protect high-priority task goals. For example, Sperandio (1978) reported that air-traffic controllers resorted to less demanding strategies to assign flight routes to pilots as the number of aircraft increased. Rather than working out which were the more efficient or comfortable routes, the controllers resorted to assigning standard routes to the aircraft. In this way, an adequate level of safety was preserved, despite the higher number of aircraft under control.

It can be assumed that the preservation of the primary task goal is also important in an everyday skill such as car driving. Arriving at the planned destination is clearly a main task goal in car driving, as is avoiding accidents. Potential disturbances are the demands of secondary tasks, such as tuning the car stereo, making telephone calls, or talking with passengers. It can be assumed that car drivers will attempt to protect the main task goal from these disturbances.

A considerable amount of research has been carried out into the effects of secondary tasks on driving performance. Some studies did find adaptive reactions of car drivers. (Note that in this paper, adaptation refers to behavioural changes aimed to protect the main task goal in high task demand situations; it should be distinguished from the concept of 'behavioural adaptation' (sometimes termed 'risk compensation'), which refers to behavioural changes specifically triggered by safety measures, such as airbags, antilock brakes, or collision avoidance systems.) For example, Jordan and Johnson (1993) showed that the time to complete a route increased when drivers had to operate the car stereo. This implies that driving speed was lower. Other studies, using direct speed measures, found that car drivers reduced their driving speed when secondary task demands increased (Pohlman and Traenkle 1994, Dingus et al. 1997, Srinivasan and Jovanis 1997). Noy (1989) found that car drivers increased their distance headway to the front car when looking at an in-car display.

On the other hand, many traffic studies report additional tasks to have negative effects on driving performance. Traffic research shows that drivers can be so distracted by subsidiary tasks that driving performance is affected. For example, Landsdown (1997) and Fairclough et al. (1993) found that drivers reduced their mirror checking, when they were driving with a visual route guidance (RG) system. The same effect was found when drivers performed an auditory memory task (Brookhuis et al. 1991). Pohlman and Traenkle (1994) found that drivers deviated from their lane more when they drove with a complex visual RG system than when driving with a common paper map. Dingus et al. (1997) also reported that drivers had more unplanned lane deviations when driving with complex RG systems due to inappropriately long glances at the displays. It has also been reported that drivers did not increase their headway to the car in front sufficiently to accommodate for the increased reaction time due to performing a secondary task, even though they did have the opportunity to do so (Alm and Nilsson 1995). Also, when drivers performed a distracter task where they had to indicate on a touch screen whether a number presented on a display matched the first number in a memory set, drivers used more time to start braking and to come to a stop (Hancock et al. 1999).

The results seem contradictory. One reason for this discrepancy might lie in the nature of the secondary tasks used in driving behaviour research: not all secondary 
tasks are irrelevant for the driver. For example, although checking a RG system and performing a memory task are both tasks additional to driving, $\mathrm{RG}$ information is highly relevant for drivers, whereas a memory task presents information that is irrelevant for the driving task. We hypothesized that tasks that serve the driving task directly will receive higher priority from drivers than tasks that are less important to the driving task. To understand which subtasks of the driving task are more important to drivers than others, it should be acknowledged that car driving is a hierarchical task, where arriving safely at the planned destination is the main task goal (Michon 1985). Behaviour at the operational level (speed control, lateral control, mirror checking) is thus under control of task goals at the tactical and strategic level. However, this top-down control does not rule out bottom-up control. Many tactical and operational task goals in driving are determined by the traffic environment, and only to a lesser extent by general task goals such as being in a hurry. For example, whether a driver will overtake depends on the traffic environment: only when there is a slow car in front is overtaking opportune. Many task goals in driving are therefore created dynamically as a response to external events, and are thus context-dependent.

People performing complex dynamic tasks can use different strategies to achieve their task goals. When faced with high task demands, operators may increase the amount of mental effort invested to ensure the same level of performance. Alternatively, they may decide to use less demanding strategies while still trying to achieve the main task goal, and in this respect, skipping a less essential subtask may help to protect the main task goal. We assume that tasks that serve goals high in the driving task hierarchy are considered more important than lower-order goals, and that tasks not directly related to the driving task are neglected more often in highdemand situations. There is indeed evidence that this is the case.

The most important way of reducing task demands for drivers is by reducing driving speed. Many studies found that drivers reduce their driving speed when task demands increase. Lower driving speeds allow more time for information processing and are thus highly effective in reducing the demands on the driver. For example, Dingus et al. (1997) used different RG information systems: visual displays, traditional paper maps, or messages by voice. They found a relation between level of demands and driving speed. The authors noted the long duration of glances at the displays of the visual RG systems, indicating high visual demands; in general, long glances are taken as evidence that the information presented is difficult to process (Fairclough et al. 1993). Interestingly, systems with the highest visual demands were associated with lowest driving speeds. The speed reduction with these systems can thus be interpreted as an adaptive response by the subjects to poor design of the system.

Other research shows that these speed reductions are indeed not random but are particularly found in situations where the demands from the RG system are highest. Pohlman and Traenkle (1994) studied the effects of different RG systems on driving performance. According to the authors, the high visual demands of the electronic and paper maps resulted in deteriorated lateral control and speed reductions without consideration of the traffic regulations or other road users, especially near intersections. Obviously, it is at intersections that drivers interact with other traffic most, so attentional demands will be highest, and perhaps more importantly, it is near intersections that route decisions have to be made: it is where the RG system will be consulted most heavily. The visual load of the RG systems will therefore be 
most prominent near intersections, and hence, speed reductions will be largest. Burnett and Joyner (1997) also found that when driving on a motor way, visual allocation to the RG system is highest when approaching an exit, which also resulted in more compensatory steering wheel movements.

Another way of reducing task demands is increasing time headway to a car in front, which allows more time to react to speed changes of the lead car. Fairclough and Graham (1999) found that drivers who had not slept for a whole night had a longer time headway to a front car than drivers who had slept for only $4 \mathrm{~h}$ and drivers who had had a normal night sleep to compensate for slower reactions. The authors interpreted this as an adaptive strategy to counteract an increased risk of rear-end collision due to the impairment caused by sleep deprivation. Van Der Hulst et al. (2001) found that after prolonged driving, drivers who reported an increase in fatigue increased their time headway, whereas drivers who did not report more fatigue after prolonged driving, did not increase time headway. This clearly suggests that fatigue influences time headway such that fatigued drivers may invest less effort while still preserving traffic safety. The same authors also reported that in limited preview conditions (fog), drivers increased their time headway to compensate for later detection of speed changes of the front car (Van Der Hulst et al. 1998).

A related finding is that drivers after prolonged driving or sleep deprivation invest less effort in their steering performance. Standard deviation of lateral position, a measure of swerving, increases as a function of time on task (Brookhuis and De Waard 1993, Van Der Hulst et al. 2001) and as a result of sleep deprivation (Fairclough and Graham 1999). Psychophysiological results from Egelund (1982), Brookhuis and De Waard (1993) and Fairclough and Graham (1999) indicate that prolonged driving results in less effort invested, and this is reflected in steering performance. Interestingly, Fairclough and Graham (1999) emphasized that partially sleep-deprived drivers only showed decrements in non-safety-critical behaviours, not in safety-critical tasks. They only neglected their steering until the situation became more critical, which was evidenced by the increased frequency of near-lane-crossings compared with the other experimental groups. The authors interpreted this minimum strategy as a compensatory response to the impairment due to sleep deprivation. Drivers who were fully deprived of sleep were however unable to protect fully safety-critical tasks, that is, they showed more lane crossings, and the number of lane crossings increased with time on task.

Thus, drivers have been shown to neglect a non-critical subtask, when their psychophysiological state is non-optimal. Dropping a non-essential subtask is also a major strategy when dealing with high task demands.

Drivers perform several subtasks during driving (e.g. McKnight and Adams (1970) described 45 major tasks and 1700 sub-tasks in driving). Many subtasks are a direct part of the driving task (e.g. speed control, steering, visual search, distance keeping), but others are not (tuning the radio, picking up the car telephone). It can therefore be assumed that car drivers safely drop certain subtasks when task demands increase. Because of the hierarchical nature of the driving task, car drivers only perform a certain type of behaviour if it serves a higher task goal. It thus depends on the priority that is assigned to the higher task goal whether a particular type of behaviour can be omitted if task demands increase. Furthermore, the same task goal can be highly relevant in one context and inappropriate in another, and the driving context thus determines to a large extent the priority of a certain task goal. 
This is exemplified by studies on visual sampling behaviour under high task demands. Checking the rear-view mirrors helps building up an overview of the traffic situation and is important when slowing down, e.g. on approach to an intersection, but it is critical when the task goal is to change lane. Giving up mirror checking under high task demands is therefore only critical to traffic safety in certain situations. A number of authors have studied rear-view mirror checking of drivers under high task demands: Landsdown (1997) used visual displays with congestion information and route guidance (RG) symbols to increase task demands; Brookhuis et al. (1991) presented subjects with a mental arithmetic task over the car telephone. These studies showed that car drivers checked their rear-view mirror less frequently when task demands increased.

This can be considered an adaptive response of the drivers to the high task demands. Apparently, in these studies, task demands increased to such an extent that drivers gave up certain aspects of their behaviour. It is very likely that subjects chose to give up the subtask of mirror checking, because in these studies it was not part of a high-priority task goal. In Landsdown's (1997) driving simulator study, the frequency of mirror checking only decreased in the RG condition: in the congestion warning condition, there was no difference compared with the control condition. Apparently, subjects' priority was more with the route guidance information than with mirror checking. This is not surprising, considering that route finding is one of the most important subgoals in driving. Furthermore traffic density was low (Landsdown 1997: 222), so the need to check the mirrors may not have been great.

The study by Brookhuis et al. (1991) was performed on the road. It included three levels of traffic density, and subjects drove with and without an auditory memory task in all traffic densities. In the condition of the highest traffic density, subjects checked their rear-view mirror less frequently than in other traffic densities. In contrast to the lowest traffic density situation, the frequency of rear-mirror checking in the other density conditions was the same for both memory task conditions; performing the memory task did not reduce mirror checking. This makes sense, since in high traffic density, monitoring other traffic is a high-priority task. The authors' explanation for this 'minimum strategy' of mirror checking was that drivers do not reduce mirror checking below a certain level. However, subjects' behaviour may have been even more strategic than this. To impose a 'heavy traffic' condition, the subjects were instructed to follow a lead car as accurately as possible. The subjects' main task was therefore to react to actions of the lead car. This instruction obviously rules out the possibility of overtaking it. Therefore, the necessity to check the mirrors was already low, irrespective of the memory condition.

Other studies on visual sampling behaviour confirm that car drivers' visual behaviour is context-dependent. De Waard (1991) compared the time that car drivers looked at traffic-relevant and traffic-irrelevant objects for different road sections. He found that on a combined entrance/exit section of a motorway, drivers spent more time looking at traffic-relevant objects (for example, other cars) compared with driving on a straight section of the road. Wierwille et al. (1991) showed that as traffic density increased, drivers looked less at the visual display on the dashboard and more at the traffic environment and observed that 'drivers do adapt reliably and appropriately to both anticipated and unanticipated increases in driving task demand while navigating'. These studies show that in demanding situations, car drivers will focus their visual attention on information relevant for the present task goal, and neglect information that is irrelevant (see also Vicente and Wang 1998). 
Previous research thus support the view that drivers protect high-priority task goals, possibly at the expense of low-priority goals in cases of high task demands. However, this assumption has not been tested directly. The present study therefore examined directly whether drivers give less priority to tasks external to the driving task than they do to tasks that serve the driving task more directly. The experiment was performed in a driving simulator, enabling the presentation of the same conditions to all subjects. As an external task we used an auditory working memory (WM) task, which had no direct relevance for the driving task of the subjects, and we assumed that subjects might neglect this task if task demands increased. In half of the conditions, subjects received simple route guidance messages; in the other half of the conditions, subjects had to use a schematic paper map of the town for route finding. We assumed that map reading is directly relevant for the driving task because it serves the main task goal in driving of arriving at the destination. We therefore hypothesised that subjects would not neglect this task, even when task demands were high. Instead, we assumed that they would reduce their driving speed to decrease the actual task demands.

\subsection{Subjects}

\section{Method}

Twenty male subjects from our institute's subject pool participated in the study. All had taken part in earlier experiments in the simulator. Mean age was 32.9 years (SD 7.3) and mean annual kilometres driven was 19000 (SD 12000). They were paid a fixed amount for their participation.

\subsection{Experimental environment and conditions}

The experiment was performed in a fixed-based driving simulator (described by Van Wolffelaar and Van Winsum 1995). The simulator car is a BMW 525 with original controls, which are all linked to the driving simulator. The pedals and the steering wheel provide resistance feedback as in a real car. The Silicon Graphics simulator has a 165 degree angle of vision horizontally and a 45 degree angle of vision vertically. The simulator software presents interactive sound of the engine depending on the number of revolutions of the engine.

Subjects drove in an urban environment (with a speed limit of $50 \mathrm{~km} / \mathrm{h}$ ) with many intersections, traffic signs, buildings, and traffic lights. There was other, artificially intelligent, traffic driving in this town, which reacted in a naturalistic way to the actions of other cars and to those of the participant. The scenario specification facility of the simulator allowed the presentation of the same traffic situations at the same locations for all participants. Almost all road sections were in built-up areas, which had the effect that subjects could only see other cars approaching an intersection when the other cars were about $30 \mathrm{~m}$ from an intersection because of buildings along the road obstructing the view.

All participants completed two journeys, one in low and one in high traffic density. In heavy traffic there were about twice as many cars at intersections and on the road. The route for the two journeys was the same, except that they were driven in opposite directions. The order of traffic conditions was balanced across subjects.

Each journey consisted of four consecutive rides, representing four conditions, in which two factors were varied: type of route guidance (RG) and presence or absence of a working memory task (WM). Participants were given two types of route guidance: they were either given a map (MAP), or they received simple auditory 
route guidance messages (ARG). The four conditions in a ride were therefore: MAP with WM task, MAP without WM task, ARG with WM task, and ARG without WM task. The order of these four conditions within a journey was balanced across subjects, but for each subject, the order of the four conditions in quiet and busy traffic was the same (e.g. if a subject started with ARG with WM task in high traffic density, he would also start with ARG with WM task in low traffic density). The total number of conditions therefore was eight (see table 1). Each condition lasted about 4 min, depending on speed driven.

In the ARG conditions, route guidance messages were given by an electronic female voice and were a simple 'turn right' or 'turn left', presented $100 \mathrm{~m}$ before an intersection. When no message was presented, subjects had to drive straight on.

In the MAP conditions, participants were given a schematic paper map, which indicated the route to drive. Road names or other landmarks were not indicated on the map as they were also absent in the simulated environment due to processing limitations of the simulator computer. All intersections and roads of the town were however clearly indicated on the map. Therefore, although the paper map was not a real road map, it provided all the necessary cues for navigating. We believe that this was not unrealistic, as people in real life sometime have to navigate in an unfamiliar environment by means of schematic drawings of the route to drive. After all, people in general may use environmental cues such as street names, shops and other landmarks while navigating, but they do not always have such information available. Also because both the simulated environment and the map were short of distracting information, we believe that the overall navigation task of subjects was probably not more demanding than on the real road.

The WM task consisted of a traffic broadcast containing six messages of incidences of traffic congestion on existing Dutch motorways. The six messages indicated road number, direction and length (e.g. A1, direction Amsterdam, $4 \mathrm{kms}$ ); the motorways chosen are well-known for their traffic congestion. The traffic broadcast occurred at a specific point in a condition, about halfway through a condition, and was announced by a short statement that traffic information would follow. The subjects' task was to add up the lengths of all the traffic queues mentioned and state this total length (ranging from 33 to $37 \mathrm{kms}$ ) at the end of a condition. The traffic broadcast lasted about $30 \mathrm{~s}$. Traffic information was presented by the same female voice that gave route guidance. If an ARG message had to be given during the traffic broadcast, the onset of a traffic message was delayed (e.g. A1, go left, direction Amsterdam, $4 \mathrm{kms})$.

If subjects lost their way, they were instructed to stop and park the car, then they were put back on the road where they had started in that condition. If subjects then lost their way again, the next condition was started.

\subsection{Procedure}

Upon arrival, the participants were given general instructions about the experiment. To allow heart rate measurement, ECG electrodes were fixed on the chests of the participants. Subjects were trained in the driving simulator for about $10 \mathrm{~min}$, or longer if required, until they were able to make smooth turns in the urban environment.

Heart rate was collected throughout the experiment; reference heart rate was collected three times for about $4 \mathrm{~min}$ (to serve as a baseline measurement): after the training, i.e. before the experiment actually started, and after the first and second 
Table 1. Overview of the eight experimental conditions

\begin{tabular}{lccccc}
\hline & \multicolumn{3}{c}{ MAP } & & \multicolumn{2}{c}{ ARG } \\
\cline { 2 - 3 } \cline { 5 - 6 } & no WM & WM & & no WM & WM \\
\hline Quiet traffic & MAP & MAP/WM & & ARG & ARG/WM \\
Busy traffic & MAP & MAP/WM & & ARG & ARG/WM \\
\hline
\end{tabular}

MAP: driving with a map; ARG: driving with auditory route guidance messages; WM: working memory task

journey. During these rest periods, subjects were requested to sit in the simulator car and relax. During these rest periods and all experimental conditions, subjects were asked not to speak, and to avoid moving unnecessarily to avoid disturbances of the ECG signal. ECG was recorded continuously during all conditions.

At the start of a condition, subjects were requested by an electronic female voice to start the engine and drive off. When driving speed was over $5 \mathrm{~m} / \mathrm{s}$, an event code was automatically generated by the driving simulator computer to mark the beginning of the condition in both the heart rate recording file and the driving parameters recording file. The end of each condition was a specific location in the ride, and when the subject arrived there, the same electronic voice asked the subject to stop and park the car on the shoulder of the road, and an event code was automatically generated to mark the end of the condition in all recording files. Immediately after a subject had parked and come to a stop, he was asked to give the total length of the traffic queues (in the WM conditions); no feedback on accuracy was given. Subjects were then requested to fill out a self-report scale for mental effort (see below). Then followed the instructions about the next condition, and when the subject was ready, the next condition was started by the auditory instruction to start the engine and drive off.

\subsection{Data collection and analysis}

After correction of heart rate, heart rate variability was calculated with CARSPAN (Mulder et al. 1988). The mid-frequency band $0.07-0.14 \mathrm{~Hz}$ (referred to as heart rate variability, HRV, from now on) was used as a measure of mental effort. Heart rate and HRV have repeatedly been shown to be sensitive to effort invested (e.g. Mulder and Mulder 1987, Wilson 1992), especially over short periods of about 3 - 5 min (e.g. Aasman et al. 1987). Heart rate can be considered a global measure of general arousal, whereas HRV can be considered a specific measure of mental effort (Wilson and Eggemeier 1991, Mulder et al. 2000), that is, mental effort involved in manipulating and maintaining information in working memory. Because of the event codes generated by the driving simulator, all heart rate data could be ascribed to each condition in a precise manner.

Averaged heart rate of a condition was expressed as a percentage relative to the preceding resting period (reference). HRV values for each condition were normalized by logarithmic transformation and expressed relative to the preceding reference rest periods; the HRV values are thus expressed as $\ln (\operatorname{task})-\ln$ (rest). This normalisation procedure allows analyses of variance on the transformed HRV values. When mental effort increases, heart rate increases and heart rate variability decreases. 
After completion of each condition, participants were asked to indicate their invested mental effort for that ride on the Rating Scale Mental Effort (RSME) (Zijlstra 1993). This is a unidimensional scale of mental effort and runs from 0 to 150. It has been shown to be sensitive to mental effort (Verwey and Veltman 1996). The main reason for choosing this scale was ease of administration and analysis.

Speed and lateral position were measured with a sample rate of $5 \mathrm{~Hz}$. Averaged driving speed and averaged standard deviation of lateral position (SDLP, a measure of swerving) were calculated for each condition. The driving measures were averaged only across those periods when subjects were actually moving, to exclude the confounding effect that waiting at traffic lights etc. would have on averaged speed. Possible collisions, near-collisions and deviations out of lane were noted.

The total length of traffic queues that the subject reported at the end of a condition was recorded as correct or incorrect. The number of navigational errors was also recorded.

All measures, except navigational errors and WM performance, were analysed by means of analysis of variance. Factors included traffic density, type of route guidance (map or ARG), WM task and the interactions between these factors.

\section{Results}

Due to simulator sickness, one participant dropped out of the study. Due to apparatus failure, driving performance data were incomplete for five participants and heart rate data for four participants. Heart rate data of one subject were excluded because they contained too many physiological artefacts (uncompensated extra-systoles) making it impossible to ascribe HRV to mental effort investment. Data for heart rate and driving performance were therefore analysed for 14 subjects. There were no main effects of order for the factor 'traffic density' for any of the variables, so this is not considered in the subsequent analyses.

\subsection{Workload measures}

Multivariate analyses of variances were performed on heart rate and normalised HRV. Driving with a map resulted in higher heart rates. This effect of type of route guidance $(\mathrm{RG})$ on $H R$ was significant $(F(1,13)=21.92, p<0.001)$. The WM task did not increase heart rate significantly $(F(1,13)=4.05, p<0.067)$. The interaction between WM and RG was also not significant $(F(1,13)=0.98$, NS). HR was not significantly affected by traffic density $(F(1,13)=1.51$, NS), nor were any of the interactions with traffic density significant. None of the effects on transformed $H R V$ values were significant.

MAP conditions were rated as requiring more mental effort than ARG conditions $(F(1,13)=17.76, p<0.002)$. The WM task also increased $R S M E$ (see figure 1) significantly $(F(1,13)=33.89, p<0.001)$. The subjective ratings also showed a significant interaction effect between $\mathrm{RG}$ and WM $(F(1,13)=7.23, p<0.016)$ : performing a WM increases effort more when driving with a map. RSME showed no significant main effect for traffic density $(F(1,13)=0.43$, NS). None of the interactions with traffic density were significant, either.

\subsection{Performance measures}

Speed (see figure 2) was significantly higher in conditions with ARG than in conditions with MAP $(F(1,13)=10.50, p<0.007)$. There was no significant difference between conditions with and without the WM task $(F(1,13)=0.00$, 


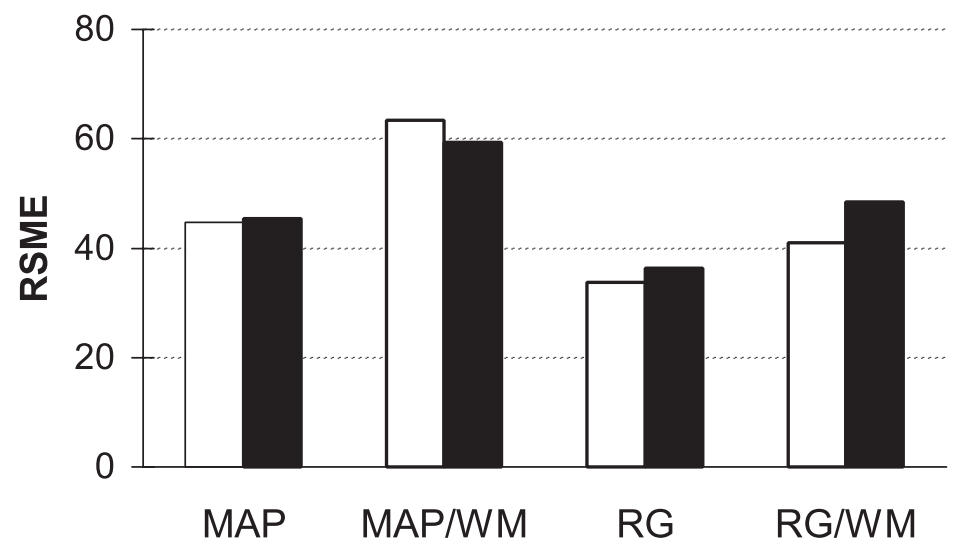

Traffic

口quiet

busy

\section{Route guidance and memory task conditions}

Figure 1. Subjective ratings of mental effort.

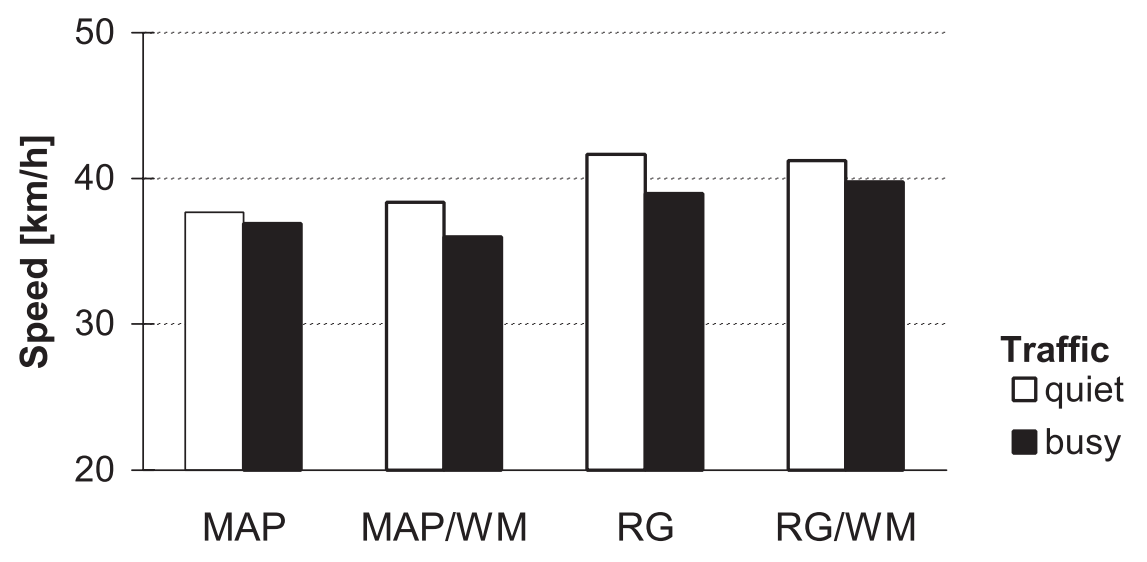

\section{Route guidance and memory task conditions}

Figure 2. Averaged driving speed.

NS), nor was the interaction between RG and WM significant. Mean speed was significantly higher in low traffic density than in high intensity $(F(1,13)=5.55$, $p<0.036$ ), but there were no significant interactions between traffic density and RG or WM.

The standard deviation of lateral position, SDLP, (see figure 3) was significantly larger (i.e. subjects swerved more) in MAP conditions, than in ARG conditions $(F(1,13)=6.88, p<0.022)$. There was no significant effect of the WM task $(F(1,13)=0.21, \mathrm{NS})$. None of the interactions were significant. SDLP showed no significant effect of traffic density $(F(1,13)=4.04, p<0.067)$.

No collisions or near-collisions were noted, nor were deviations out of lane.

Working memory performance varied considerably between conditions. In MAP conditions, the percentage of correct answers (see table 2) was consistently lower 


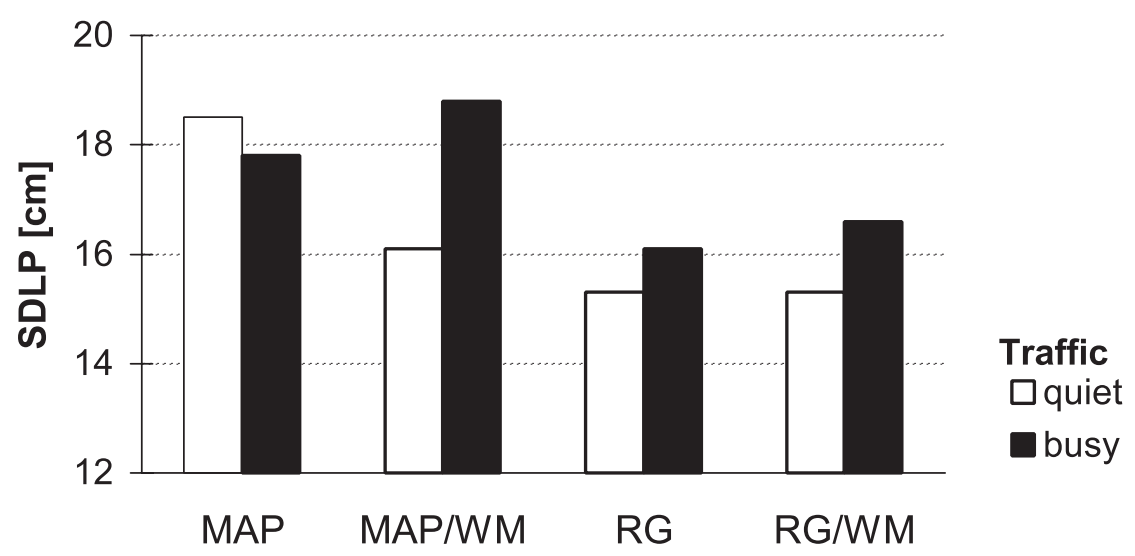

Route guidance and memory task conditions

Figure 3. Averaged standard deviation of lateral position (SDLP).

Table 2. Memory task performance: percentage of subjects correct

\begin{tabular}{lcc}
\hline & MAP & ARG \\
\hline Quiet traffic & 26 & 74 \\
Busy traffic & 47 & 63 \\
\hline
\end{tabular}

MAP: driving with a map; ARG: driving with auditory route guidance messages

than in ARG conditions, with less than half of the subjects giving correct answers. Only $74 \%$ of the subjects gave the correct answer in the easiest condition, which showed that this task was a difficult one.

No subject made a navigational error (see table 3 ) with route guidance (ARG) without the WM task and only $5 \%$ of the subjects when ARG was combined with the WM task. However, in the MAP conditions, this percentage increased to $42 \%$ in quiet traffic with the WM task. Unexpectedly, more subjects lost their way in quiet than in busy traffic.

\section{Discussion}

Under high task demands, drivers may not be able to perform all the tasks. We hypothesized that in such conditions, drivers do not drop tasks randomly but will try to protect high-priority tasks. More specifically, we assumed that drivers will protect tasks directly related to the main driving task. Before discussing the results, it may be useful to examine how different methods of prioritising might lead to different results.

If drivers prioritize their tasks in view of the relevance of the tasks, than the effects of increased task demands would be different for different tasks. Tasks related to the main goal in driving (to arrive safely at the destination) should be protected against performance degradation in high task demands, for example by reducing driving speed. Low-priority tasks, not relevant for the main driving task, might receive less 
Table 3. Route errors: percentage of subjects taking a wrong turn

\begin{tabular}{lccccc}
\hline & \multicolumn{3}{c}{ MAP } & & \multicolumn{2}{c}{ ARG } \\
\cline { 2 - 3 } \cline { 5 - 6 } & no WM & WM & & no WM & WM \\
\hline Quiet traffic & 26 & 42 & & 0 & 5 \\
Busy traffic & 16 & 16 & & 0 & 5 \\
\hline
\end{tabular}

MAP: driving with a map ; ARG: driving with auditory route guidance messages; WM: working memory task

attention in high task demands, and performance on that task may consequently suffer.

In the present experiment, we assumed that map reading was a high-priority task because it served the main objective to arrive at the destination. We therefore expected that navigating with a map would be compensated for by reducing driving speed, resulting in equal number of route errors in all conditions. The WM task was designed to be a task of low priority (it was irrelevant for the driving task) and we expected that subjects might pay less attention to the WM task when task demands were high, resulting in worse WM task performance in MAP conditions and in busy traffic. Because drivers would not be motivated to protect this task, we expected that subjects would not reduce their driving speed when they performed the WM task.

High traffic density represents an increase in primary task demands that subjects cannot neglect in the same way as a secondary task can be neglected. We therefore expected that in high traffic density driving speed would be lower than in low traffic conditions as a way to compensate for the increase in primary task demands.

In sum, if our hypothesis is correct, driving speeds would be reduced in busy traffic and in MAP conditions, but not in WM conditions. Route errors would occur in equal amounts in all conditions, but the number of correct answers on the WM task would be the lower in high task demand conditions, i.e., when driving with a map and in busy traffic. If our hypothesis is not correct, than driving speeds would be reduced in busy traffic, in MAP and in WM conditions as drivers will have no preference to protect one specific task. Route errors would occur in equal amounts in all conditions, and the number of correct answers on the WM task would be the same across conditions.

Let's first examine whether we were successful in increasing task demands. Workload measures indeed showed that the experimental manipulations increased task demands. Subjects' rating of mental effort increased from about 35 ('some effort') in the easiest condition (driving with ARG), to about 60 ('rather much effort') in the most effortful condition (map reading combined with performing the WM task). Heart rate followed a similar pattern: it increased when subjects drove with a map and when the WM task was performed, and was highest in the combined condition (MAP/WM).

Heart rate variability (HRV) showed a different pattern: there were no significant effects of any of the experimental manipulations, which was quite an unexpected finding. HRV has repeatedly been shown to be specifically sensitive to increases in mental effort, not only in general laboratory and applied settings (for a review, see Wilson and Eggemeier 1991, see also Mulder et al. 2000), but also more specifically in driver behaviour studies (e.g. De Waard et al. 1994). We believe that subjects' 
behavioural strategies reduced task demands to such an extent that mental effort was not significantly increased in any of the conditions. Below we will show why this is a plausible explanation.

\subsection{General findings}

The main finding was that subjects reduced their driving speed in busy traffic, and when navigating with a map, but not when performing the WM task. The fact that driving speed was reduced when driving with a map shows that navigation was considered an important task, while the lack of speed reduction in WM conditions show that our subjects did not consider the WM to be a task of high priority that has to be protected at any cost. This is strong support for our hypothesis that drivers prioritize their tasks with respect to their relevance to the main driving task.

Route errors were however not distributed equally across conditions, as we had hypothesized. More route errors were made when subjects used the paper map rather than listening to simple ARG messages. Moreover, navigational performance suffered from concurrent performance of the WM task, and it was worse in quiet rather than busy traffic. This is contrary to our expectations. WM task performance was largely reduced when driving with a map, which is in accordance with our hypothesis. However, WM task performance were unexpectedly better in busy than in quiet traffic.

Thus, while the results for driving speed were as expected, the effects of traffic density on secondary task performance were unexpected. What might explain these results? We believe that the reduced driving speed in heavy traffic may account for this. We will discuss this in the next sections, where we will first discuss the effects of traffic density on driving speed more thoroughly before discussing secondary task performance.

\subsection{The effects of traffic density on driving speed}

As stated above, driving speed was reduced in high traffic density compared to low traffic density. It should be noted that driving speed in heavy traffic was not lower simply because subjects waited longer at intersections to give priority to more cars. Driving speed was averaged only for periods in which subjects were actually driving, and therefore, periods where subjects were waiting were not used to calculate averaged driving speed. However, more traffic on the road reduces averaged driving speed indirectly because in heavy traffic there were more intersections with other cars present, and at those intersections, there were more cars. Subjects thus had to wait more often and for longer periods at intersections. Therefore, there were more instances of slowing to a stop and accelerating after a stop, which obviously reduces averaged driving speed.

However, driving speed was also lower as a result of subjects' strategies: when driving with a map, they seemed to approach intersections at lower speeds, possibly because this allowed them more time to attend to the more complex traffic situation at the intersection, to look longer at the map and listen to the WM task. In fact, we noted that in MAP conditions, subjects also seemed to wait longer before accelerating, and it appeared to us that they did this especially to be able to look longer at the map. In sum, waiting more often at intersections contributed to lower averaged driving speed in busy traffic, while the increased length of these waits also increased the time available to consult the map (or listen to the WM task). 


\subsection{The effects of type of route guidance}

4.3.1. Driving performance: Map reading required drivers to take their eyes off the road, and drivers reduced their driving speed to compensate for this. This is in accordance with our hypothesis about map reading being a high-priority task that drivers try to protect. Another effect of driving with a map was that subjects swerved more. In itself, this is not surprising: any task which requires drivers to take their eyes off the road while driving will increase swerving, for the simple reason that visual information is needed for course keeping. However, subjects had already reduced their driving speed to counteract this negative effect of map reading, but apparently this was not sufficiently to abolish an increase in swerving completely.

However, we do not believe that this indicates that drivers were in fact neglecting the main driving task when driving with a map. First, in the present experiment map reading was part of the main driving task, and swerving more might actually be seen as evidence for the high priority assigned to it. Second, subjects tried to minimize interference between the conflicting task goals of course keeping and route finding. Rather than checking the map while driving, subjects primarily checked their map when they were waiting for other traffic at intersections, and as noted, they actually seemed to wait longer in busy traffic before accelerating. This strategy largely prevents having to look at the map when driving although the increase in swerving indicates that this strategy did not preclude it completely.

Interestingly, the strategy to consult the map when waiting at the intersection may also explain the lack of effects of driving with a map on HRV. It indicates that subjects probably did not memorise the complete route at the beginning of each condition. Instead, they probably cut the route into smaller chunks ('I have to turn left at second crossing') and then, at each intersection when standing still, subjects updated their information ('Yes, I have to turn right at this intersection'). This strategy largely reduces working memory use, and thereby the amount of mental effort that is needed for this task. This strategy may explain why HRV showed no effect of map use. The significant change in averaged heart rate and in self-reported effort in MAP conditions showed that subjects were more generally aroused and investing more effort when driving with a map than when following auditory route instructions, suggesting that map use increased task demands significantly.

Third, we believe it unrealistic to assume that the main goal in driving is precise course keeping, that is, to keep lateral position as constant as possible. Minor deviations from a certain criterion in lateral and longitudinal control are not necessarily detrimental to safe driving. It should be pointed out that the increase in SDLP, although significant, amounted to only a few centimetres, and this does not indicate a significant threat to traffic safety. In addition, no deviations out of lane were noted. Therefore, it seems justified to state that when navigating with a map, subjects did not neglect the main driving task completely.

4.3.2. Route errors: Contrary to our expectations, route errors were not distributed equally across conditions. They were predominately made when subjects had to find their route by using a paper map and were virtually absent when simple ARG messages told subjects their way. This suggests that the simple ARG messages were quite easy to follow, while map reading was a difficult task to perform. It also indicates that subjects' reduction in driving speed did not fully compensate for these high task demands. 
A counterintuitive finding was that more route errors were made in quiet traffic than in busy traffic. In both conditions in busy traffic, $16 \%$ of the participants took a wrong turning; however, in quiet traffic, these percentages were 26 and 42 in MAP and MAP/WM conditions. As noted before, this is probably due to subjects driving slower and waiting longer at intersections in high traffic density. This gave subjects more time to consult the map and thus helped them significantly in finding their route on the map.

These data confirm our hypothesis that drivers consider route finding an important subtask of driving, and it suggests that they might even find it more important than precise course keeping. Subjects were confronted with two conflicting subgoals: course keeping and map reading. Together, the decrease in driving speed, the increase in SDLP, and the large amount of route errors in some conditions, show that our subjects were not entirely able to resolve the conflict between the two task goals.

\subsection{Performing a working memory task while driving}

In contrast to our predictions, performing the working memory task while driving was not associated with a decrease in HRV, which would have indicated more mental effort. Not only is this in contrast to our own expectations, it is also in contrast to other similar traffic research. For example, in the study by Brookhuis et al. (1991), subjects also had to perform a mental arithmetic task while driving. In that study, HRV was reduced compared to driving without the MA task.

What might explain the lack of effect on mental effort (HRV) is the relatively poor performance on the WM task. In the easiest condition, 74\% of the subjects gave the correct answer (ARG, quiet traffic), but this number dropped to $26 \%$ in the MAP condition in quiet traffic. Together with the fact that HRV shows no mental effort on the memory task, these performance data strongly suggest that the subjects did not give much attention to the WM task in more demanding conditions. Especially in the MAP conditions, where only a minority of the subjects gave the correct answer, subjects did not invest much effort into the WM task.

The finding that the WM task was performed better in busy rather than in quiet traffic was surprising, but it can probably be explained by the longer waits at intersections in busy traffic. It should be noted that the WM task only lasted about $100 \mathrm{~s}$, and waiting for, say, $20 \mathrm{~s}$ longer at an intersection when the WM task is presented increases significantly the time available to listen to this task without driving. Unfortunately, we took only an aggregate measure of WM performance (naming the longest traffic queue at the end of the ride) rather than monitoring task performance continuously, so we cannot ascertain this assumption directly.

Putting it all together, there as thus two reasons why secondary task performance was better in busy traffic. First, driving speed was lower, allowing more time to look at the map (although driving speed was not lower in WM task conditions). Second, subjects had to wait more often and for longer periods at intersections. The longer waits at intersections allowed drivers more time to consult the map and listen to the WM task resulting in better WM task and route finding performance.

\subsection{General conclusions}

Self-reported effort (RSME) showed that, in some conditions, our subjects had to invest considerable effort to deal with the task. This is also clear from the increase in heart rate in more demanding conditions (MAP, WM task). This indicates not only 
that our subjects were actively involved in the task, but also that the demands on the subjects were high. Actually, our subjects were not able to perform all the (sub)tasks, as shown by the relatively poor performance on the working memory and the navigational task, and by the increase in swerving when subjects navigated with a map. This demonstrates that the combined task demands were clearly beyond the capabilities or motivation of our subjects.

Hockey (1997) stated that operators can basically do two things when task demands increase: invest more effort, or adopt less strict performance targets to protect the high-priority task goal, at the expense of subsidiary activities. Our results support this view: performance on the subsidiary tasks suffered most from the high task demands. Hockey (1997) calls this latent performance decrement. Our subjects indeed seemed to regulate their own task demands, either by reducing driving speed or by taking a more lenient performance criterion on less important tasks. The present data suggest something more: different sources in increases in task demands may have differential effects on behavioural strategies.

The working memory task is an artificial task without clear relevance to the main task goal in driving. Performing the WM task means an increase in secondary task demands. When traffic density increased or subjects had to read the map, subjects dealt with these further increases in task demands by reducing their efforts in the artificial WM task. In other words, subjects (partly) gave up secondary task performance when they can or will not perform an additional task on top of the primary task.

In contrast, an increase in traffic density implies an increase in primary task demands. Subjects cannot neglect this in the same way as they can neglect performing a secondary task. In our experiment, subjects reduced their driving speed to reduce the demands. This means that they changed the way in which the main task was performed. As an additional effect, reduced driving speed resulted in better WM task and navigational performance. This can be interpreted as secondary effects of the main strategy to protect the main task.

Although map reading may be considered a task additional to the main driving task, route finding is in fact an important subtask in an unfamiliar environment. As map reading implies taking the eyes off the road, map reading hampers precise course keeping, and it thus produced a conflict between the subgoals of route finding and course keeping in the present study. Results show that subjects were unable to resolve this conflict. This was evidenced both by the increase in swerving and the many route errors when subjects drove with a map. The fact that subjects swerved more with a map may indicate that navigation was indeed considered an important subtask, and was even performed at the expense of precise course keeping.

These date support the hypothesis by Hockey (1997) that task operators will protect high-priority task goals. Car drivers seem to protect their primary task from degradation due to secondary task intrusion by remaining focused on the driving task, at the expense of secondary task performance. The data also indicate that map reading may not be a secondary task in the same way as an external (working memory) task is. Route finding is an important subtask for drivers, and the data suggest that it may be at conflict with course keeping.

Lastly, it is important to note that 'deteriorated' performance on a secondary task thus does not mean that the operator's performance is inadequate. Rather, the operator has enhanced the efficiency of his task behaviour. It may allow more time or more mental capacity to perform other (sub)tasks, which may be more relevant 
for the present context, or to perform better on the primary task. It is important to realise that the performing operator determines the relevance of a specific (sub)goal, not the observing researcher. Care should be taken that a particular change in task behaviour is not interpreted as a deterioration in task performance. Such a change probably serves a (sub)goal deemed important by the operator. The challenge to the researcher lies in finding out which goal is served by such a change.

4.5.1. Practical implications: It thus appears that drivers can ignore some tasks, such as placing a mobile phone call, or perhaps checking the rear-view mirrors in certain situations. Other additional tasks, most notably route finding, are probably more important to drivers. The present experiment implies that this should have major implications for the design of route guidance systems in particular. More than most other in-car equipment, RG systems present information that is highly important to drivers in unfamiliar environments, and car drivers will be highly motivated to check the RG system, even in difficult traffic situations. Ironically, it is especially near intersections that the need for route information is high. This places high demands on an adequate design of RG systems, and they should be designed in such a way that visual demands are reduced as best as possible.

\section{References}

Alm, H. and Nilsson, L. 1995, The effects of a mobile telephone task on driver behaviour in a car following situation, Accident Analysis and Prevention, 27, 707-715.

Aasman, J., Mulder, G. and Mulder, L. J. M. 1987, Operator effort and the measurement of heart-rate variability, Human Factors, 29, 161-170.

Bainbridge, L. 1974, Problems in the assessment of mental load, Le Travail Human, 37, 279 302.

Bainbridge, L. 1997, The change in concepts needed to account for human behaviour in complex dynamic tasks. IEEE Transactions on Systems, Man, and Cybernetics-Part A: Systems and Humans, 27, 351-359.

Brookhuis, K. A., De Vries, G. and De WaArd, D. 1991, The effects of mobile telephoning on driving performance, Accident Analysis and Prevention, 23, 309-316.

Brookhuis, K. A. and De WaARD, D. 1993, The use of psychophysiology to assess driver status. Ergonomics, 36, 1099-1110.

Bruckmayer, E., Becker, S., Brockman, M., Erkan, S., Mertens, A., Mihm, J. and Sontag, J. 1995, Evaluation of the STORM route guidance system by in-car observation using an instrumented vehicle, HOPES Report 27, Köln: Technische Überwachungsverein.

Burnett, G. and Joyner, S. 1997, An assessment of moving map and symbol-based route guidance systems, in Y. I. Noy (ed.), Ergonomics and Safety of Intelligent Driver Interfaces. Human Factors in Transportation (Mahwah, NJ: Lawrence Erlbaum Associates), 115-137.

DE WAARD, D. 1991, Driving behaviour on a high-accident-rate motorway in the Netherlands, in C. Weikert, K. A. Brookhuis and S. Ovinius (eds), Man in Complex Systems. Proceedings of the Human Factors Society Annual Meeting (Work Science Bulletin 7). (Lund: Work Science Division, Department of Psychology, Lund University), 113-123.

De Watrd, D., Van der Hulst, M. and Brookhuis, K. A. 1994, Behavior comparator prototype test in a driving simulator, Report 2009/DETER/Deliverable 10, Traffic Research Centre, University of Groningen.

Dingus, T. A., Hulse, M. C., Mollenhauer, M. A., Fleischman, R. N., McGehee, D. V. and MANAKKal, N. 1997, Effects of age, system experience, and navigation technique on driving with an advanced traveler information system, Human Factors, 39, 177-199.

Egelund, N. 1982, Spectral analysis of heart rate variability as an indicator of driver fatigue, Ergonomics, 25, 663-672. 
Fairclough, S. H., Ashby, M. C. and Parkes, A. M. 1993, In-vehicle displays, visual workload and usability evaluation, in A. G. Gale, I. D. Brown, C. M. Haslegrave, H. W. Kruysse and S. P. Taylor (eds), Vision in Vehicles-IV. (Amsterdam: Elsevier), 245-254.

Fairclough, S. H. and Graham, R. 1999, Impairment of driving performance caused by sleep deprivation or alcohol: a comparative study, Human Factors, 41, 118-128.

Hancock, P. A., Simmons, L., Hashemi, L., Howarth, H. and Ranney, T. 1999, The effects of in-vehicle distraction on driver response during a crucial driving maneuver, Transportation Human Factors, 1, 295-309.

Hockey, G. R. J. 1997, Compensatory control in the regulation of human performance under stress and high workload: a cognitive-energetical approach, Biological Psychology, 45, $73-93$.

Hockey, G. R. J., Wastell, D. G. and Sauer, J. 1998, Effects of sleep deprivation and user interface on complex performance: a multilevel analysis of compensatory control, Human Factors, 40, 233 - 253.

Jordan, P. W. and Johnson, G. I. 1993, Exploring mental workload via TLX: The case of operating a car stereo whilst driving, in A. G. Gale, I. D. Brown, C. M. Haslegrave, H. W. Kruysse and S. P. Taylor (eds), Vision in Vehicles-IV (Amsterdam: Elsevier), $225-$ 262.

Landsdown, T. C. 1997, Visual allocation and the availability of driver information, in T. Rothengatter and E. Carbonell Vaya (eds), Traffic and Transport Psychology: Theory and Application (Oxford: Pergamon), 215-223.

McKnight, A. J. and Adams, B. B. 1970, Driver education task analysis. Volume 1: Task descriptions, Final report, Contract No FH 11-7336, Alexandria, VA: Human Resources Research Organization.

Michon, J. A. 1985, A critical view of driver behavior models: what do we know, what should we do?, in L. Evans and R. C. Schwing (eds), Human Behavior and Traffic Safety (New York: Plenum Press), 485-524.

Mulder, L. J. M. and Mulder, G. 1987, Cardiovascular reactivity and mental work-load, in R. I. Kitney and O. Rompelman (eds), The Beat-to-beat Investigation of Cardiovascular Function: Measurement, Analysis and Application (Oxford: Clarendon Press), 216-253.

Mulder, G., Mulder, L. J. M., Meijman, T. F., Veldman, J. B. P. and Van Roon, A. M. 2000, A psychophysiological approach to working conditions, in R. W. Backs and W. Boucsein (eds), Engineering Psychophysiology: Issues and Applications (Mahwah, NJ, USA: Lawrence Erlbaum Associates). 139-159.

Mulder, L. J. M., Van Dellen, H. J., Van der Meulen, P. and Opheikens, B. 1988, CARSPAN, A spectral analysis program for cardiovascular time series, in F. J. Maarse, L. J. M. Mulder, W. Sjouw and A. Akkerman (eds), Computers in Psychology: Methods, Instrumentation and Psychodiagnostics (Lisse: Swets \& Zeitlinger), 30-38.

Noy, Y. I. 1989, Intelligent route guidance: Will the new horse be as good as the old?, Proceedings of the Vehicle Navigation and Information System Conference VNIS, (Toronto), 49-55.

Pohlmann, S. and Traenkle, U. 1994, Orientation in road traffic. Age-related differences using an in-vehicle navigation system and a conventional map, Accident Analysis and Prevention, 26, 689-702.

Sperandio, J. C. 1978, The regulation of working methods as a function of work-load among air traffic controllers, Ergonomics, 21, 195-202.

Srinivasan, R. and Jovanis, P. P. 1997, Effect of in-vehicle route guidance systems on driver workload and choice of driving speed: findings from a driving simulator, in Y. I. Noy (eds), Ergonomics and Safety of Intelligent Driver Interfaces. Human Factors in Transportation. (Mahwah, NJ: Lawrence Erlbaum Associates), 97-114.

Van Der Hulst, M., Meijman, T. and Rothengatter, T. 2001, Maintaining task set under fatigue: a study of time-on-task effects in simulated driving, Transportation ResearchPart F: Psychology and Behaviour, 4, 103-118.

Van der Hulst, M., Rothengatter, T. and Meijman, T. 1998, Strategic adaptations to lack of preview in driving. Transportation Research-Part F: Psychology and Behaviour, 1, $59-$ 75. 
Van Wolffelaar, P. C. and Van Winsum, W. 1995, Traffic modelling and driving simulationan integrated approach, in Conference Proceedings of Driving Simulation Conference (DSC'95). (Toulouse, France: Teknea), 236-244.

Verwey, W. B. and Veltman, H. A. 1996, Detecting short periods of elevated workload: A comparison of nine workload assessment techniques, Journal of Experimental Psychology: Applied, 23, 270-285.

Vicente, K. J. and WANG, J. H. 1998, An ecological theory of expertise effects in memory recall, Psychological Review, 105, 33-57.

Welford, A. T. 1978, Mental work-load and a function of demand, capacity and skill, Ergonomics, 21, $151-167$.

Wierwille, W. W., Hulse, M. C., Fischer, T. J. and Dingus, T. A. 1991, Visual adaptation of the driver to high-demand driving situations while navigating with an in-car navigation system, in A. G. Gale, I. D. Brown, C. M. Haslegrave, I. Moorhead, and S. Taylor (eds), Vision in Vehicles-III. (Amsterdam: Elsevier), 79-87.

WiLson, G. F. 1992, Applied use of cardiac and respiratory measures: practical considerations and precautions, Biological Psychology, 34, 163-178.

Wilson, G. F. and Eggemeier, F. T. 1991, Physiological assessment of workload in multi-task environments, in D. L. Damos (eds), Multiple-task Performance. (London: Taylor \& Francis), 329-360.

Zislstra, F. R. H. 1993, Efficiency in work behaviour: a design approach for modern tools, doctoral dissertation, Delft University Press, Delft. 